\title{
Droplet-based microfluidics for high-throughput screening of a metagenomic library for isolation of microbial enzymes
}

Masahito Hosokawa $^{\mathrm{a}, \mathrm{b}, \mathrm{c}}$, Yuri Hoshino ${ }^{\mathrm{a}}$, Yohei Nishikawa ${ }^{\mathrm{a}}$, Tomotada Hirose ${ }^{\mathrm{d}}$, Dong Hyun Yoon ${ }^{\mathrm{d}}$, Tetsushi Mori $^{\mathrm{a}, \mathrm{b}, \mathrm{c}}$, Tetsushi Sekiguchi ${ }^{\mathrm{b}}$, Shuichi Shoji ${ }^{\mathrm{b}, \mathrm{d}}$, and Haruko Takeyama ${ }^{\mathrm{a}, \mathrm{b}, \mathrm{c}_{*}}$

a Department of Life Science and Medical Bioscience, Waseda University, 2-2 Wakamatsu-cho, Shinjuku-ku, Tokyo 162-8480, Japan

b Institute for Nanoscience and Nanotechnology, Waseda University, 513, Wasedatsurumaki-cho, Shinjuku-ku, Tokyo 162-0041, Japan

c Core Research for Evolutionary Science and Technology (CREST), Japan Science and Technology Agency (JST), 5, Sanbancho, Chiyoda-ku, Tokyo 102-0075, Japan

d Faculty of Science and Engineering, Waseda University, 3-4-1, Okubo, Shinjuku-ku, Tokyo, 169-8555, Japan

* Author to whom correspondence should be addressed; E-Mail: haruko-takeyama@waseda.jp; Tel./Fax: +81-3-5369-7326. 


\section{Abstract}

This paper proposes a high-throughput, function-based screening approach of a metagenomic library for isolating novel microbial enzymes by droplet-based microfluidics. We used gel microdroplets (GMDs) dispersed in oil as picoliter-volume reaction vessels for lipolytic enzyme by encapsulating cells in individual GMDs. Using this approach, we monitored the growth of individual cells encapsulated in GMDs and assessed the enzyme reaction activities at the level of an individual GMD. We then applied this method to screen lipolytic enzyme genes from the metagenomic library constructed from soil collected from a quercus serrate forest of Mount Tsukuba, Ibaraki, Japan. In the workflow presented in this study, metagenomic library clones were encapsulated in 100-pL GMDs with a fluorogenic reporter substrate. A total of 67,000 metagenomic library clones can be screened in only $24 \mathrm{~h}$ with reduced consumption of reagents (i.e., $<10 \mu \mathrm{L}$ ). As a result, we identified a novel lipolytic enzyme, EstT1, belonging to the EstD2 family of esterases and containing a putative signal peptide, which facilitates enzyme export and catalyzation of substrates in the periplasm. Our study demonstrates the potential of microfluidic GMDs as an efficient tool for metagenomic library screening of industrially relevant enzymes with the potential of significantly reducing the cost and time factors involved in successful practical application of microbial enzymes.

\section{Keywords}

droplet; microfluidics; metagenomic library; single-cell analysis; lipolytic enzyme 


\section{Introduction}

Enzymes find use in a wide range of applications in industries as environmentally benign catalysts. Environmental microbes have received much attention as a potential source of industrially relevant enzymes (Hasan et al., 2006). However, the standard laboratory culture techniques support the growth of $<1 \%$ of the environmental bacteria (Amann et al., 1995). Thus, as a cultivation-independent approach, metagenomics has served as an effective tool for the screening of candidate enzymes for industrial use from both cultured and uncultured microorganisms (Daniel, 2005; Lee and Lee, 2013; Lorenz and Eck, 2005; Simon and Daniel, 2011). In this metagenomics approach, microbial DNAs, which are directly extracted from the environmental samples such as soil, are cloned in a surrogate host to enable exhaustive investigation of microbial DNA fragments independent of the culturability of the source microbes. In general, metagenome libraries are screened based on either the sequence or function. In sequence-based screening, direct sequencing of metagenomic DNA has recently been performed using next-generation sequencing techniques to search for microbial enzymes and bioactive compounds from the microbial community (Banik and Brady, 2008; Dinsdale et al., 2008; Teeling and Glockner, 2012). However, this approach is limited to the detection of gene variants containing the conserved motif from the metagenome and may not allow screening of novel genes. On the other hand, function-based screening is a straightforward approach to obtain novel enzyme genes (Uchiyama and Miyazaki, 2009). In this case, the enzyme activities are assayed by cultivating a metagenomic library on agar plates or microtiter plates supplemented with substrates, following the identification of positive clones through visual 
screening for the appearance of a clear zone called a halo or through colorimetric reaction. However, conventional function-based screening of metagenomic libraries face some challenges such as a low hit rate of positive clones, labor intensiveness, low throughput, and excess time consumption.

The gel microdroplet (GMD) technique has been used in various bacterial studies, including for improvement of culturability of the environmental bacteria and isolation of individual microorganisms (Dichosa et al., 2014; Fitzsimons et al., 2013; Katsuragi et al., 2000; Manome et al., 2001). Compartmentalization of cells within GMDs provides a sequestered microenvironment facilitating the growth of single cells, microcolony formation, and accumulation of biological reaction products. The droplet technology combined with fluorescent-activated cell sorting (FACS) allows high-throughput screening of enzyme libraries with fluorogenic substrates (Aharoni et al., 2005). Although the conventional bulk emulsification technique for generation of droplets shows large variance in the droplet diameter, the recent droplet-based microfluidics approach has greatly improved the variance and reproducibility of generation of nano- to femtoliter-sized droplets (Joensson and Andersson Svahn, 2012; Theberge et al., 2010). Droplet-based microfluidic is recognized as an efficient tool for the assessment of cellular behavior at the single-cell level by co-encapsulating cells producing target molecules (e.g., enzymes and antibodies) with a reporter molecule such as a fluorescent substance (Agresti et al., 2010; He et al., 2005; Najah et al., 2012; Scanlon et al., 2013; Sjostrom et al., 2014) or microbeads for capturing the secreted products (Mazutis et al., 2013). Compartmentalization of cells in surfactant-stabilized droplets surrounded by immiscible oil can be used to isolate individual reaction vessels, eliminating the risk of 
cross-contamination and promoting uniform mixing of the reagents inside the droplets. (Baret, 2012).

Furthermore, it enables high-throughput screening of producer cells in digital format, based on the presence or absence of reporter products in the droplets (Baret et al., 2009; Joensson et al., 2009; Leng et al., 2010; Schmitz et al., 2009; Shim et al., 2009).

In this study, we applied droplet-based microfluidics for function-based screening of a metagenome library constructed from soil samples to isolate novel lipolytic enzymes, including lipase (EC 3.1.1.3) and esterase (EC 3.1.1.1). Lipolytic enzymes are often used for various industrial applications such as synthesis of ester compounds for pharmaceuticals, foods, detergents, and other biochemical purposes (Rogalska et al., 1997). To date, various lipolytic-active enzymes have been isolated from different metagenomic libraries in various environments (Daniel, 2005; Lorenz and Eck, 2005). We previously demonstrated the screening of a novel lipolytic enzyme from a metagenome library constructed from marine sponges (Okamura et al., 2010). However, the limited throughput of function-based screening continues to remain an issue. Therefore, in this paper, we present a microfluidic GMD technique for co-encapsulation of metagenomic clones with a fluorogenic substrate for function-based screening of a metagenomic library based on a lipolytic activity assay (Fig. 1). Following the growth of the host, which was encapsulated at the single-cell level, and the induction of the recombinant expression cassette, GMDs containing cells producing lipolytic enzymes are distinguished by the presence of fluorescent products. We validated our method by using a lipase-expressed Escherichia coli model and demonstrated our method by screening a soil metagenomic library for lipolytic enzyme. The metagenomic fosmid libraries of soil 
collected from the forest in Mount Tsukuba were assessed by using our microfluidic GMDs. Consequently, we isolated a novel lipolytic enzyme, EstT1, showing similarity to the recently discovered EstD2 family of lipolytic enzymes (Lee et al., 2010). Our results demonstrate the potential of microfluidic GMDs as an efficient tool for screening a metagenomic library for industrially relevant enzymes along with great reduction in the cost and time investment required for the practical application of microbial enzymes.

\section{Materials and methods}

\subsection{Metagenomic library construction}

Soil samples were collected from a quercus serrate forest situated in Mount Tsukuba (Tsukuba, Ibaraki, Japan). Soil DNA was isolated and purified from $10 \mathrm{~g}$ of soil sample by using ISOIL (Nippon Gene; Tokyo, Japan), according to the manufacturer's protocol. A fosmid library of extracted DNA was constructed by using the CopyControl ${ }^{\mathrm{TM}}$ Fosmid Library Production Kit, according to the manufacturer's protocol with small modifications reported by Gurgui and Piel, (Gurgui and Piel, 2010). DNA fragments were purified, ligated into the pCC1FOS fosmid vector, and subsequently packaged into lambda phages by using MaxPlax Lambda Packaging Extracts (Epicentre; Madison, WI). The packaged library was transduced into the EPI300-T1 ${ }^{\mathrm{R}}$ E. coli cells, which were then spread onto Luria Bertani (LB) agar supplemented with $12.5 \mu \mathrm{g} / \mathrm{mL}$ chloramphenicol. Based on the titer of the phage particles calculated by a colony counting assay, the library clones were stored in tubes as library pools containing 1000 clones $/ \mathrm{mL}$ of $50 \%$ glycerol at 
$-80^{\circ} \mathrm{C}$ until screening for active clones. As a lipolytic active control, we constructed E. coli cells expressing lipase, which was exported into the periplasm. The plasmid pET22b (+) containing the gene for lipase (lip32Nc) was kindly provided from Suntory Holdings Limited (Osaka, Japan). The plasmid was used to transform E. coli BL21 (DE3). Lipase activities of the transformants were confirmed by halo formation on LB agar containing ampicillin, $100 \quad \mu \mathrm{M}$ isopropyl- $\beta$-D-thiogalactopyranoside (IPTG), and $1 \%$ tributyrin, and the selected transformants were used for the following experiments.

\subsection{Cell sample preparation}

In cell sample preparation for cell-encapsulation experiments, the LB medium and yeast extract peptone dextrose (YPD) medium were prepared for preculturing of E. coli and Saccharomyces cerevisiae K7 cells, respectively. After overnight culturing of E. coli and yeast cells, the cell concentrations of each medium were calculated and then diluted with dispersed phase liquids. For evaluating the numbers of encapsulated cells in GMDs, the cells were fixed with paraformaldehyde and then stained with propidium iodide (PI) prior to diluting with the dispersed phase liquids.

\subsection{Fabrication of microfluidic droplet generator}

In this study, a flow-focusing microfluidic device was used to generate agarose GMDs (Shah et al., 2008). In this device, a cell suspension containing ultra-low gelling temperature agarose was pumped into the cross-junction as a dispersed phase liquid, while the immiscible carrier phase liquid 
is driven from the other inlet. These two phases meet at the cross-junction and the droplets were periodically pinched off from the dispersed phase (Fig. 2a). The cross-junction microfluidic droplet generator was fabricated by the photolithography technique. The droplet generator was designed by AutoCAD (AutoDesk; Sausalito, CA), and the corresponding photomasks were printed (Toyo Precision Parts Mfg. Co., Ltd.; Nara, Japan). The mask pattern was transferred onto a layer of negative photoresist (SU8-3050; Microchem; Newton, MA) coated on glass wafer $(40 \times 49 \mathrm{~mm})$, and master mold for 50- $\mu$ m-deep microfluidic channels was fabricated. All the microchannels were $50-\mu \mathrm{m}$ tall and $400-\mu \mathrm{m}$ wide, except at the cross-junction area. The cross junction was designed with 30-, 50-, and 100- $\mu \mathrm{m}$ width from the upstream to the downstream region, gradually (Fig. 2a). Poly(dimethylsiloxane) (PDMS; Silpot 184: Dow Corning Toray Co., Ltd.; Tokyo, Japan) and a cross-linker were mixed thoroughly at the ratio of 10:1 (w/w). The degassed PDMS mixture was poured over the mastermold and coated onto a glass slide $(40 \times 49 \mathrm{~mm})$ by a spin coater, followed by curing for at least $30 \mathrm{~min}$ at $70^{\circ} \mathrm{C}$. Upon curing, the PDMS slabs were carefully peeled off the molds. The PDMS slabs and PDMS-coated glass slides were bonded by a plasma treatment (Aiplasma; Panasonic Corporation; Osaka, Japan), followed by baking for at least $30 \mathrm{~min}$ at $70^{\circ} \mathrm{C}$.

\subsection{Emulsification of single microbial cells}

As dispersed phase liquids, LB medium and yeast extract malt extract (YEME) medium containing ultra-low gelling temperature agarose (Sigma-Aldrich; St Louis, MO) at a concentration of 1.0\% (w/v) was prepared for E. coli and S. cerevisiae, respectively. As the continuous oil phase, two kinds 
of oil were prepared: mineral oil (Sigma-Aldrich) containing 3\% (v/v) of the surfactant Span80 (Sigma-Aldrich) and fluorinated oil (FC40; Sigma-Aldrich) containing 2\% (v/v) of the surfactant Pico-Surf1 (Dolomite; Charleston, MA). The surfactant stabilizes the droplets and prevents coalescence. For single-cell encapsulation in GMDs, the E. coli or S. cerevisiae cell suspension was diluted in each medium immediately prior to droplet generation to the final concentration of $1.3 \times$ $10^{4}$ cells $/ \mu \mathrm{L}$ for mineral oil and $8.8 \times 10^{3}$ cells $/ \mu \mathrm{L}$ for fluorinated oil. The cell suspension and carrier oil were loaded into a 500- $\mu \mathrm{L}$ Hamilton gas-tight syringe (Hamilton; Reno, NV) connected to Tefzel $^{\circledR}$ tubing (0.8-mm ID), which in turn was connected to the inlets of the PDMS device. The device outlet was also connected to a 1.5-mL Eppendorf-type tube via Tefzel ${ }^{\circledR}$ tubing (0.2-mm ID). Droplets were generated by introducing both the cell suspension and carrier oil into the microfluidic droplet generator by using the KDS-210 syringe pumps (KD Scientific; Hillston, MA). The flow rate was controlled at $60 \mu \mathrm{L} / \mathrm{h}$ for agarose solution, $180 \mu \mathrm{L} / \mathrm{h}$ for mineral oil, or $600 \mu \mathrm{L} / \mathrm{h}$ for fluorinated oil. The process of droplet generation was monitored under a fluorescence microscope (IX71; Olympus Corporation; Tokyo, Japan) integrated with a high-speed camera (Fastcam-neo $32 \mathrm{~K}$; Photoron; Tokyo, Japan). The droplets were collected and cooled on an ice bath for 15 min for agarose gelation to form GMDs. The formed GMDs were incubated at $37^{\circ} \mathrm{C}$ for E. coli cells and $30^{\circ} \mathrm{C}$ for yeast cells in the tubes and then transferred into capillary tubes (Vitrocom; Mountain Lakes, NJ) for microscopic observation (Fig. 2b). The number and growth of encapsulated cells within the droplet were evaluated with bright or fluorescent images obtained by using a fluorescence 
microscope (BX51; Olympus Corporation) integrated with a digital camera (DP-73; Olympus Corporation).

\subsection{Lipolytic enzyme activity assay}

In order to evaluate the lipolytic enzyme activity for metagenome screening, the commercially available lipase or $E$. coli cells harboring a plasmid for the expression of lipase in the periplasm were encapsulated in GMDs generated from LB medium containing ampicillin, fluorogenic substrate (50 $\mu \mathrm{M}$ fluorescein dicaprylate), IPTG, and 1\% agarose. To evaluate the lipolytic enzyme activity based on fluorescence measurement, we used the commercially available enzyme substrate fluorescein dicaprylate, which does not fluoresce by itself. The microdroplets were incubated at $37^{\circ} \mathrm{C}$ for $24 \mathrm{~h}$ to facilitate cell growth and substrate hydrolysis by the enzyme. Fluorescence images of the microdroplets were captured by using the BX51 fluorescence microscope integrated with a DP-73 digital camera or the IN Cell Analyzer 2000 (GE Healthcare; Tokyo, Japan). The Lumina Vision acquisition software (Mitani Corporation; Tokyo, Japan) was used to acquire and analyze the fluorescence microscopy images. Image analysis was performed to determine the lipolytic enzyme activity and to count the objects that satisfied the predetermined criteria. The fluorescence intensities and morphometric characteristics such as droplet size and fluorescent intensities were considered when identifying the positive droplets.

\subsection{Screening of a soil metagenomic library for lipolytic enzymes}


The lipolytic-active clones of the soil metagenomic library were screened using the lipolytic enzyme activity assay described in Section 2.5. Metagenomic library clones were directly introduced into the droplet generator from the pools and encapsulated in GMDs at the concentration of 1 cell/GMD. The GMDs were generated in LB medium containing $1 \%$ agarose, $12.5 \mu \mathrm{g} / \mathrm{mL}$ chloramphenicol, $50 \mu \mathrm{M}$ fluorescein dicaprylate, and $1 \mathrm{X}$ CopyControl Induction solution to allow expression of metagenomic DNA. The fluorescence of GMDs observed by fluorescent microscope identified the pools containing lipolytic-active clones, based on the presence of fluorescent GMDs in whole GMDs containing single clones of the metagenomic library.

To confirm the presence of lipolytic-active clones with a conventional function-based screening method, same metagenome library was also cultured by LB agar medium emulsified with $1 \%$ tributyrin as previously described (Okamura et al., 2010). After cultivation of the library clones at $37^{\circ} \mathrm{C}$ for $2-3$ days, the clones demonstrating lipolytic activity were identified by the presence of a halo around the colony and then selected. Then, the selected clones were further tested and confirmed for their ability to hydrolyze fluorescein dicaprylate by the GMD assay. The clones exhibiting lipolytic activity were sequenced on the 454 GS Junior instrument (Roche; Branford, CT) to determine the regions harboring the lipolytic enzyme genes. The amino acid sequences of the predicted proteins were compared with those of known lipolytic enzymes by the BLASTp program (http://www.blast.ncbi.nlm.nih.gov/). The sequence homology regions were identified through multiple alignments with the ClustalW program. The phylogenetic tree was constructed by the unweighted pair group average linked method using arithmetic averages (UPGMA). 
2.7. Nucleotide sequence accession number

The nucleotide sequence of $e s t T 1$ gene has been assigned GenBank accession number AB948213. 


\section{Results and Discussion}

\subsection{Single-cell encapsulation in GMD}

In general, the sizes of generated droplets depend on the geometry of the microchannel of flow-focusing microfluidic device, the flow rate of the aqueous and oil phases, and the viscosities of the two phases. First, we optimized the droplet-generation condition with the flow rate of the cell suspension kept constant to prevent the settlement and adsorption of cells onto the microchannels. By applying two solutions at the optimized flow rates, the droplets were generated at a typical frequency of 250 droplets/sec. As the carrier-phase liquid for droplet generation, we used fluorinated oil (FC40; 3M) supplemented with 2\% Pico-surf or mineral oil supplemented with 3\% Span80. In both cases, monodispersed GMDs of diameter $59.3 \pm 1.7 \mu \mathrm{m}(109 \mathrm{pL})$ for fluorinated oil and $51.5 \pm$ $1.4 \mu \mathrm{m}(72 \mathrm{pL})$ for mineral oil were stably formed (Fig. 2b, c). On the other hand, conventional bulk emulsification generated highly polydispersed GMDs $(25.1 \pm 6.5 \mu \mathrm{m}$, coefficient of variation, $26 \%)$. Next, we evaluated the efficiency of single-cell encapsulation in GMDs by the microfluidic droplet generator. The cell encapsulation can be approximated as discrete independent events that occur randomly with time. As a result, the number of cells per GMD followed a Poisson distribution when E. coli cells were introduced at the theoretical concentration of 1 cell/GMD (Fig. 2d). Under this condition, approximately $43 \%$ of a GMD was empty, while approximately $42 \%$ contained a single $E$. coli cell after encapsulation by the microfluidic droplet generator. In addition, the gelation of agarose stabilizes the formation of droplets under cell growth temperatures and enables recovery of GMDs from oil phase by simple centrifugation and washing. The stability of the droplets is useful 
for the additional staining of cells within the GMDs for the assessment of cellular activity and the subsequent sorting of specific GMDs by FACS (Eun et al., 2011; Scanlon et al., 2013).

\subsection{Monitoring of cell activity in GMDs}

To demonstrate the capability of performing time-lapse measurements using the GMDs, we monitored the growth of cells encapsulated within the GMDs. E. coli and S. cerevisiae cells that were encapsulated in GMDs supplemented with nutrients were incubated at appropriate temperatures. The incubation of GMDs containing single E. coli (Fig. 3a) or S. cerevisiae (Fig. 3b) cells led to the formation of microcolonies with respect to time. The budding event from single yeast could be tracked by using GMDs. This feature can be applied for monitoring the pedigree of single cells and for the evaluation of dynamics and heterogeneity of cellular behaviors (Osada et al., 2014; Pan et al., 2011).

After confirmation of cell division in GMDs, we evaluated the activity of enzymes produced by the encapsulated cells. Firstly, the commercially available lipase was co-encapsulated with fluorescein dicaprylate and then incubated. During incubation, lipase catalyzes the hydrolysis of fluorescein dicaprylate to release the green fluorophore fluorescein (Fig. 4a). When using mineral oil as a carrier phase, the fluorescein is exchanged among GMDs through phase partitioning (data not shown). Therefore, we used fluorinated oil, in which the nonfluorinated compounds are highly insoluble, as a carrier phase to prevent transportation of fluorescein. To validate the assay workflow, E. coli cells expressing lipase, which was exported into the periplasm, were co-encapsulated with fluorescein 
dicaprylate in GMDs as a model, and the time-dependent change in the fluorescent intensity of GMDs was monitored for $24 \mathrm{~h}$. Following incubation, the fluorogenic substrate was diffused into the periplasm and enzymatically hydrolyzed, resulting in the spread of the fluorescent product throughout the GMDs (Fig. 4b). On the other hand, no obvious signal was observed from GMDs encapsulating E. coli harboring the control vector (data not shown). The time-dependent change of fluorescence intensities of GMDs (Fig. 4c) showed a 5-h lag that could be ascribed to the growth of the cells before the start of expression, exportation of lipase into the periplasm, and maturation of the enzyme. As a result, the fluorescence intensities of individual GMDs were gradually increased and then reached a plateau after $8 \mathrm{~h}$ of incubation. The fluorescence intensities varied significantly at the level of individual GMDs encapsulating E. coli harboring lipase genes. We considered this variation to be attributed by the initial number of encapsulated cells and the cell-cycle asynchrony as well as other factors contributing to cellular heterogeneity. The percentage of fluorescent-positive droplets $(61 \%)$ is correlated with the theoretical percentage of droplets containing one or more cells, based on the Poisson distribution. Although the droplet size should be optimized for targeted cell and enzyme, we considered that $100 \mathrm{pL} \mathrm{GMD} \mathrm{could} \mathrm{provide} \mathrm{appropriate} \mathrm{environment} \mathrm{for} \mathrm{microcolony} \mathrm{formation} \mathrm{of}$ E. coli and lipolytic enzyme reaction with reduction of reagent consumption toward metagenome library screening.

\subsection{Screening of lipolytic enzyme genes from the soil metagenomic library}


The constructed soil metagenome library contained 67,000 clones, the majority of which contained average inserts $36 \mathrm{~kb}$ in size. The whole library contained 2,412 Mb of total sequences. In this study, one of the library pools was used to validate the feasibility of our screening method. In this analysis, fluorescence was detected in 69 of 19,589 GMDs. As described above, as approximately $43 \%$ of a GMD is empty, approximately $1.1 \times 10^{4}$ cells were considered to be distributed in GMDs, resulting in an estimated hit rate of $0.62 \%$. The clones in the library pool showing the presence of fluorescent GMDs were also cultured on an agar plate containing $1 \%$ tributyrin to form fatty acid salts. After 3 days of incubation, ten positive clones generating a halo around colonies on the agar plate were identified from a total of 3527 colonies, giving a hit rate of $0.28 \%$ (Fig. S1). The positive clones were re-encapsulated to verify their ability for hydrolysis of fluorescein dicaprylate within all cell-encapsulated GMDs, and the clones were archived. We believe that the difference of hit rate between the microfluidic GMD assay and the conventional agar-plate assay can be attributed to the differences in the culture conditions such as the densities of cells, expressed enzymes, and reporter molecules. In particular, the fluorescent signals in the microfluidic GMD assay could be identified more clear and certain than the halo in the conventional agar plate assay. This advantage might mainly contribute to the difference in hit rate. The reagent volume required for the GMD-based screening (approximately $100 \mathrm{pL} / \mathrm{cell}$ ) is significantly less than that for the conventional agar-plate screening or microtiter screening assay (approximately 25-100 $\mu \mathrm{L} /$ cell). In addition, the fluorescent-microscopic screening of positive clones in the microfluidic GMDs allows better distinguishability within a short time as compared to the visual screening for the appearance of a 
halo on the agar plate. The concentrating effect of the secreted enzymes and substrates in the GMDs suggested an increase in the sensitivity of the enzyme activity assay as compared to that in the bulk assay. Indeed, the incubation time required to assess the activity of clones of a metagenomic library can be reduced from 2-3 days to $12 \mathrm{~h}$ by using the proposed GMD technique.

Sequencing the respective metagenomic inserts of the clones revealed that all lipolytic-active clones containing a putative open reading frame (ORF) shared $58.6 \%$ sequence identity with the hypothetical protein (YP_002129569) from Phenylobacterium zucineum HLK1, which was reannotated as a putative esterase in a recent study (Lee et al., 2010) (Fig. S2). This gene was designated est 1 with the accession number AB948213. To assess how this ORF is related to other known lipolytic enzymes, the phylogenetic relationship was analyzed based on the esterase/lipase classification (Arpigny and Jaeger, 1999), including recently reported families (Fig. S3). EstT1 clustered together with EstD2 isolated from a metagenomic library of plant rhizosphere soil (Lee et al., 2010). Alignment of the EstT1 sequence with the EstD2 family revealed the presence of a pentapeptide G-H-S-Q-G motif containing the active site serine 200 (Fig. S2). In addition, a signal peptide (Met1-Ala34) was identified at the N-terminus region of EstT1 by the SignalP 4.1 Server (Petersen et al., 2011), suggesting that EstT1 is exported to the periplasm and functions outside the cell. We considered that the hydrolysis in the periplasm or outside the cell could be detected in the GMDs, according to the function of this signal peptide. This screening procedure would be useful for screening periplasmically expressed enzymes and secreted enzymes (Shim et al., 2009). These 
results suggest that our microfluidic GMD technology can contribute to efficient metagenomic screening for the isolation of novel enzymes from an environmental microbial community.

\section{Conclusions}

In this study, we developed a microfluidic GMD generation method for metagenomic screening of environmental samples for the selection of of lipolytic enzymes. This system enabled function-based screening of the library, based on the results of the digital enzymatic activity assay at the single-cell level. The encapsulated cells express lipolytic enzymes that catalyze the hydrolysis of fluorescein dicaprylate, and the fluorescent products are accumulated in GMD as a function of time. As a result, the novel lipolytic enzyme EstT1 was isolated from the soil metagenomic library using this function-based screening technique and sequence analysis. This method could be applied not only to lipolytic enzymes but also to various other enzymes by using appropriate fluorogenic substrates. As described in previous reports (Agresti et al., 2010), the droplet technique has the potential for reducing the reagent consumption and the time required for high-throughput screening by using a microtiter plate robot system. Thus, the function-based screening of metagenomic libraries by using microfluidic GMDs would be a useful strategy for isolating novel enzymes from various microbial communities representing diverse environments.

\section{Acknowledgments}


This work is partly supported by JST-CREST "Establishment of core technology for the preservation and regeneration of marine biodiversity and ecosystems", MEXT Grant-in-Aid for Scientific Basic Research (S) No. 23226010, and Grant-in-Aid for Young Scientists (B) No.26820365. 


\section{Figure legends}

Fig. 1. A schematic of function-based screening of a metagenomic library for the isolation of lipolytic enzymes by using microfluidic GMDs.

The clones of the metagenomic library constructed from environment sample were encapsulated in agarose GMD with fluorogeneic substrates on a microfluidic droplet generator. After incubation, microscopic observation enabled detection and counting of lipolytic-active clones from the library pools in a digital format, based on the presence or absence of green fluorescence products in the GMDs. For characterization of the detected active clones, enzyme genes can be isolated, sequenced, cloned, recombined, or rescreened to facilitate metagenomic screening of industrially relevant enzyme genes.

\section{Fig. 2. Generation of agarose gel microdroplet (GMD) by microfluidic droplet generator.}

(a) Microphotograph of droplet generation at the microfluidic cross-junction. The cells pre-suspended in medium containing $1 \%$ agarose and fluorogenic substrates were introduced into the microfluidic device for encapsulation within GMDs at the single-cell level. Scale bar; $200 \mu \mathrm{m}$. (b) Microphotograph of $1 \%$ agarose GMD generated by microfluidic droplet generator. Scale bar; 200 $\mu \mathrm{m}$. (c) Size distribution of GMDs. GMDs were generated by introduction of LB medium containing $1 \%$ agarose as a sample phase and mineral oil containing 3\% Span80 or FC-40 containing 2\% Pico-Surf1. (d) The numbers of droplets containing different numbers of cells. E. coli cells were 
encapsulated in $1 \%$ agarose GMD at a concentration of 1 cell/GMD. Theoretical values were calculated based on the Poisson distribution.

\section{Fig. 3. Growth of microbes in microfluidic GMDs.}

(a) Microcolony formation of single E. coli cells encapsulated in 1\% agarose GMDs. Images show the cells before and after cultivation in GMDs. Arrowheads indicate cells in GMDs. Scale bar, 50 $\mu \mathrm{m}$. (b) Sequential images of $S$. cerevisiae cells encapsulated in 1\% agarose GMDs.

Fig. 4. Lipase activity assay using single cell-encapsulated GMDs.

(a) Fluorescent microphotographs of agarose GMDs containing fluorescent dicaprylate with or without purified lipase after $18 \mathrm{~h}$ of incubation. Scale bar; $100 \mu \mathrm{m}$. (b) Sequence fluorescence images of GMDs co-encapsulating E. coli cells harboring lipase genes with fluorescent dicaprylate. The cells were encapsulated in GMDs at the single-cell level and incubated for $24 \mathrm{~h}$. (c) Time-dependent change of fluorescence intensity of GMDs encapsulating E. coli cells harboring lipase genes with fluorescent dicaprylate. 


\section{References}

Agresti, J.J., Antipov, E., Abate, A.R., Ahn, K., Rowat, A.C., Baret, J.C., Marquez, M., Klibanov, A.M., Griffiths, A.D., Weitz, D.A., 2010. Proc. Natl. Acad. Sci. U. S. A. 107, 4004-4009.

Aharoni, A., Amitai, G., Bernath, K., Magdassi, S., Tawfik, D.S., 2005. Chem. Biol. 12, 1281-1289.

Amann, R.I., Ludwig, W., Schleifer, K.H., 1995. Microbiol. Rev. 59, 143-169.

Arpigny, J.L., Jaeger, K.E., 1999. Biochem. J. 343 Pt 1, 177-183.

Banik, J.J., Brady, S.F., 2008. Proc. Natl. Acad. Sci. U. S. A. 105, 17273-17277.

Baret, J.C., 2012. Lab Chip 12, 422-433.

Baret, J.C., Miller, O.J., Taly, V., Ryckelynck, M., El-Harrak, A., Frenz, L., Rick, C., Samuels, M.L., Hutchison, J.B., Agresti, J.J., Link, D.R., Weitz, D.A., Griffiths, A.D., 2009. Lab Chip 9, 1850-1858.

Daniel, R., 2005. Nat. Rev. Microbiol. 3, 470-478.

Dichosa, A.E., Daughton, A.R., Reitenga, K.G., Fitzsimons, M.S., Han, C.S., 2014. Nat. Protoc. 9, 608-621.

Dinsdale, E.A., Edwards, R.A., Hall, D., Angly, F., Breitbart, M., Brulc, J.M., Furlan, M., Desnues, C., Haynes, M., Li, L., McDaniel, L., Moran, M.A., Nelson, K.E., Nilsson, C., Olson, R., Paul, J., Brito, B.R., Ruan, Y., Swan, B.K., Stevens, R., Valentine, D.L., Thurber, R.V., Wegley, L., White, B.A., Rohwer, F., 2008. Nature 452, 629-632.

Eun, Y.J., Utada, A.S., Copeland, M.F., Takeuchi, S., Weibel, D.B., 2011. ACS Chem. Biol. 6, 260-266.

Fitzsimons, M.S., Novotny, M., Lo, C.C., Dichosa, A.E., Yee-Greenbaum, J.L., Snook, J.P., Gu, W., Chertkov, O., Davenport, K.W., McMurry, K., Reitenga, K.G., Daughton, A.R., He, J., Johnson, S.L., Gleasner, C.D., Wills, P.L., Parson-Quintana, B., Chain, P.S., Detter, J.C., Lasken, R.S., Han, C.S., 2013. Genome Res. 23, 878-888.

Gurgui, C., Piel, J., 2010. Methods Mol. Biol. 668, 247-264.

Hasan, F., Shah, A.A., Hameed, A., 2006. Enzyme Microb. Technol. 39, 235-251.

He, M., Edgar, J.S., Jeffries, G.D., Lorenz, R.M., Shelby, J.P., Chiu, D.T., 2005. Anal. Chem. 77, 1539-1544.

Joensson, H.N., Andersson Svahn, H., 2012. Angew. Chem. Int. Ed. Engl. 51, 12176-12192.

Joensson, H.N., Samuels, M.L., Brouzes, E.R., Medkova, M., Uhlen, M., Link, D.R., Andersson-Svahn, H., 2009. Angew. Chem. Int. Ed. Engl. 48, 2518-2521.

Katsuragi, T., Tanaka, S., Nagahiro, S., Tani, Y., 2000. J. Microbiol. Methods 42, 81-86.

Lee, M.H., Hong, K.S., Malhotra, S., Park, J.H., Hwang, E.C., Choi, H.K., Kim, Y.S., Tao, W., Lee, S.W., 2010. Appl. Microbiol. Biotechnol. 88, 1125-1134.

Lee, M.H., Lee, S.W., 2013. Genomics Inform. 11, 114-120.

Leng, X., Zhang, W., Wang, C., Cui, L., Yang, C.J., 2010. Lab Chip 10, 2841-2843.

Lorenz, P., Eck, J., 2005. Nat. Rev. Microbiol. 3, 510-516.

Manome, A., Zhang, H., Tani, Y., Katsuragi, T., Kurane, R., Tsuchida, T., 2001. FEMS Microbiol. Lett. 197, 29-33. 
Mazutis, L., Gilbert, J., Ung, W.L., Weitz, D.A., Griffiths, A.D., Heyman, J.A., 2013. Nat. Protoc. 8, 870-891.

Najah, M., Griffiths, A.D., Ryckelynck, M., 2012. Anal. Chem. 84, 1202-1209.

Okamura, Y., Kimura, T., Yokouchi, H., Meneses-Osorio, M., Katoh, M., Matsunaga, T., Takeyama, H., 2010. Mar. Biotechnol (NY) 12, 395-402.

Osada, K., Hosokawa, M., Yoshino, T., Tanaka, T., 2014. The Analyst 139, 425-430.

Pan, J., Stephenson, A.L., Kazamia, E., Huck, W.T., Dennis, J.S., Smith, A.G., Abell, C., 2011.

Integr. Biol. (Camb) 3, 1043-1051.

Petersen, T.N., Brunak, S., von Heijne, G., Nielsen, H., 2011. Nat. Methods 8, 785-786.

Rogalska, E., Douchet, I., Verger, R., 1997. Biochem. Soc. Trans. 25, 161-164.

Scanlon, T.C., Dostal, S.M., Griswold, K.E., 2013. Biotechnol. Bioeng.

Schmitz, C.H., Rowat, A.C., Koster, S., Weitz, D.A., 2009. Lab Chip 9, 44-49.

Shah, R.K., Shum, H.C., Rowat, A.C., Lee, D., Agresti, J.J., Utada, A.S., Chu, L.-Y., Kim, J.-W., Fernandez-Nieves, A., Martinez, C.J., 2008. Mater. Today 11, 18-27.

Shim, J.U., Olguin, L.F., Whyte, G., Scott, D., Babtie, A., Abell, C., Huck, W.T., Hollfelder, F., 2009. J. Am. Chem. Soc. 131, 15251-15256.

Simon, C., Daniel, R., 2011. Appl. Environ. Microbiol. 77, 1153-1161.

Sjostrom, S.L., Bai, Y., Huang, M., Liu, Z., Nielsen, J., Joensson, H.N., Andersson Svahn, H., 2014. Lab Chip 14, 806-813.

Teeling, H., Glockner, F.O., 2012. Brief. Bioinform. 13, 728-742.

Theberge, A.B., Courtois, F., Schaerli, Y., Fischlechner, M., Abell, C., Hollfelder, F., Huck, W.T., 2010. Angew. Chem. Int. Ed. Engl. 49, 5846-5868.

Uchiyama, T., Miyazaki, K., 2009. Curr. Opin. Biotechnol. 20, 616-622. 
Figures

Figure 1

Construction of metagenomic library from environment sample

Microfluidic GMD generation

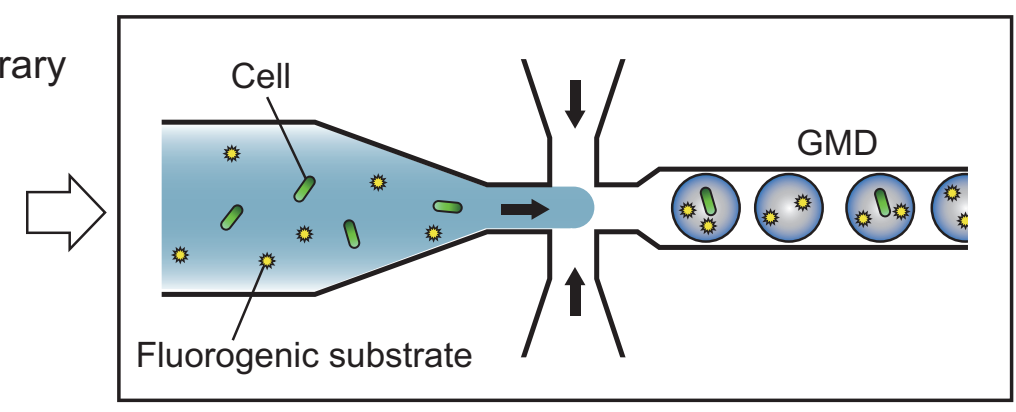

Characterization of positive clones

Digital enzymatic activity assay

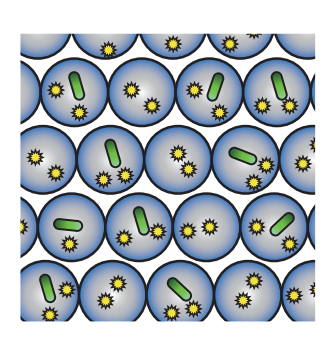

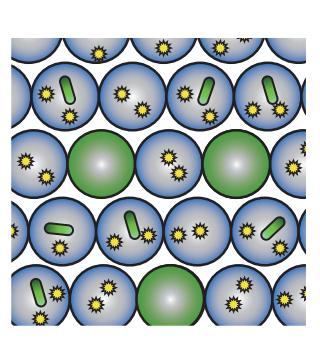

Sequencing

of metagenomic insert

Positive clone 
a

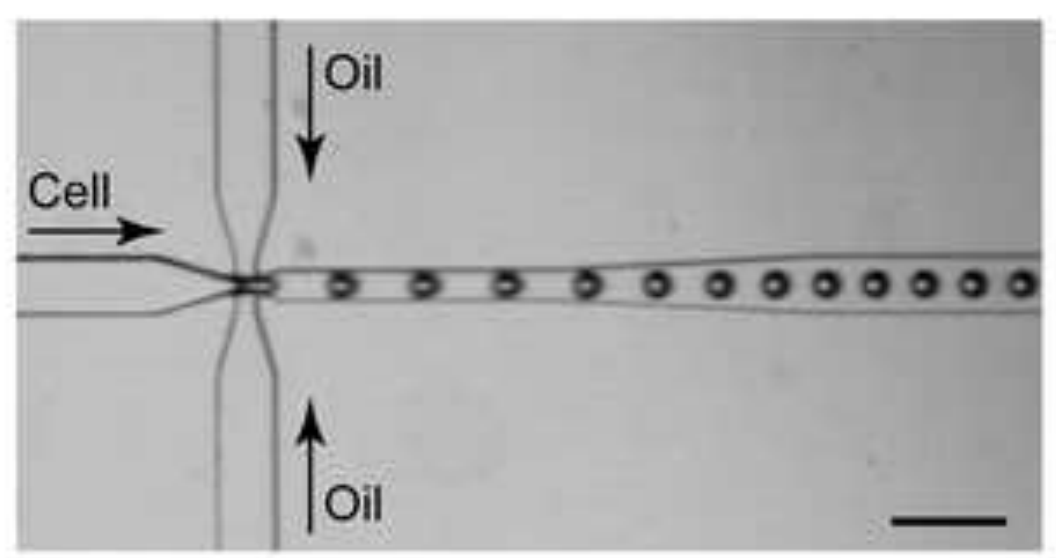

C

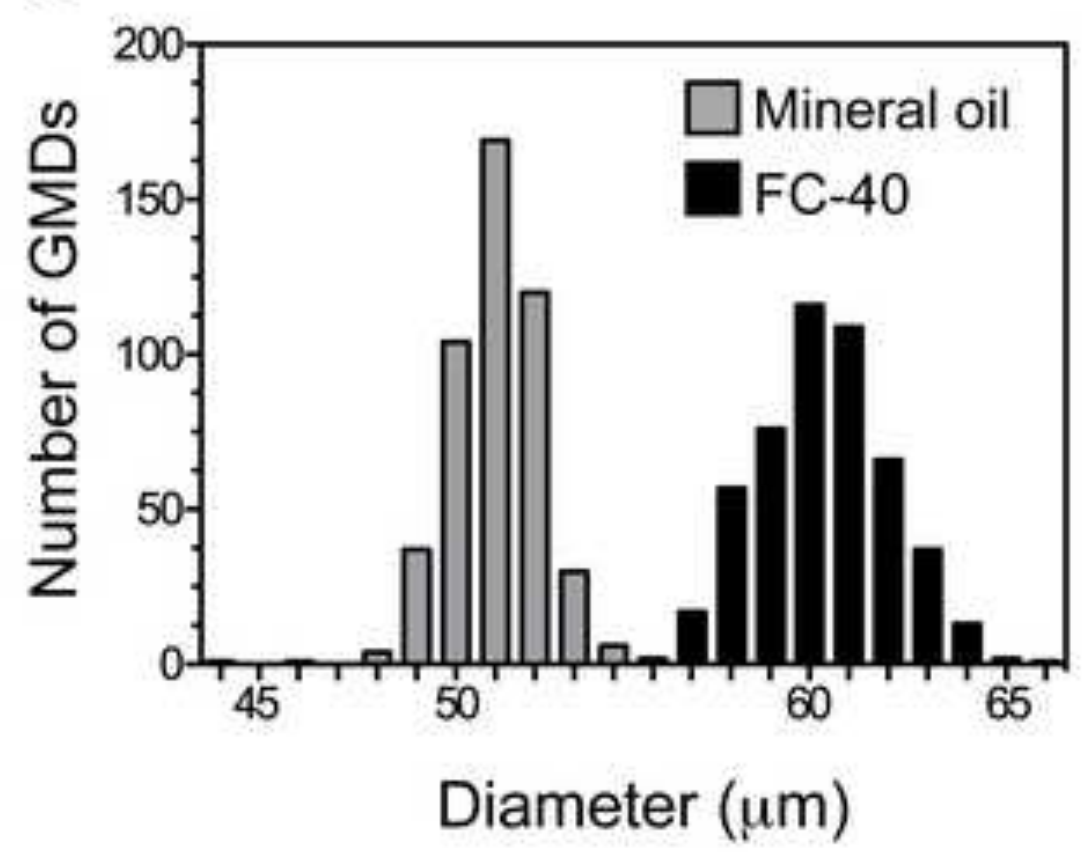

b

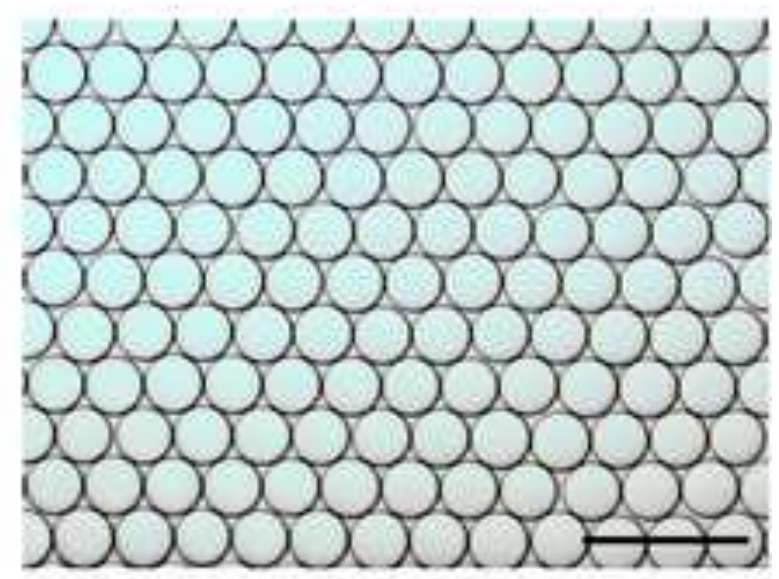

d

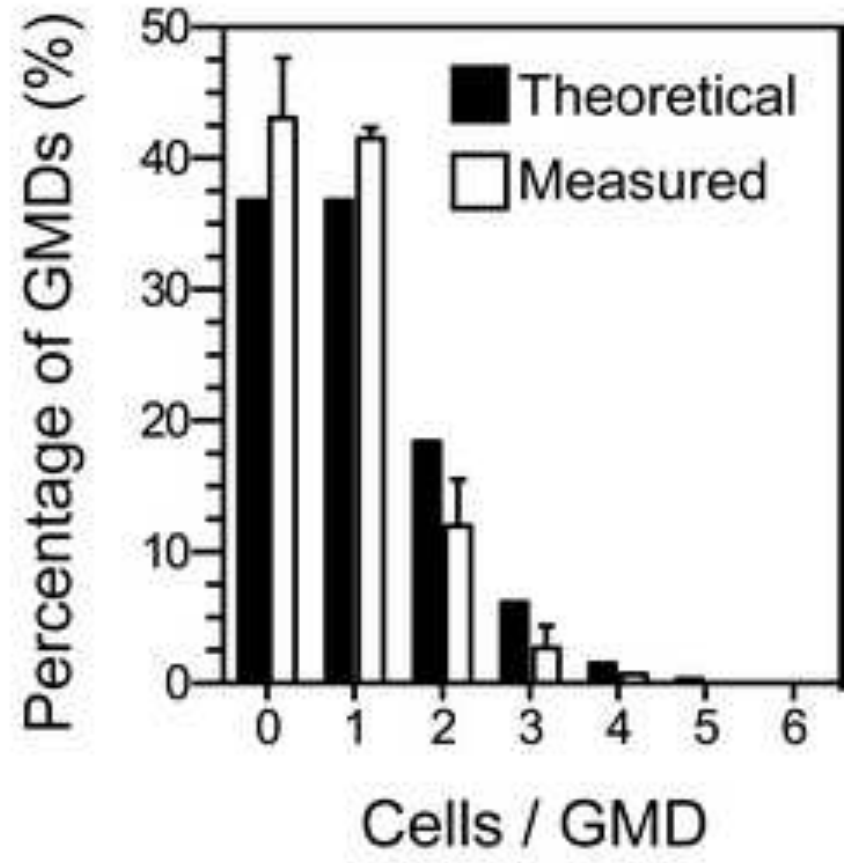


Figure 3
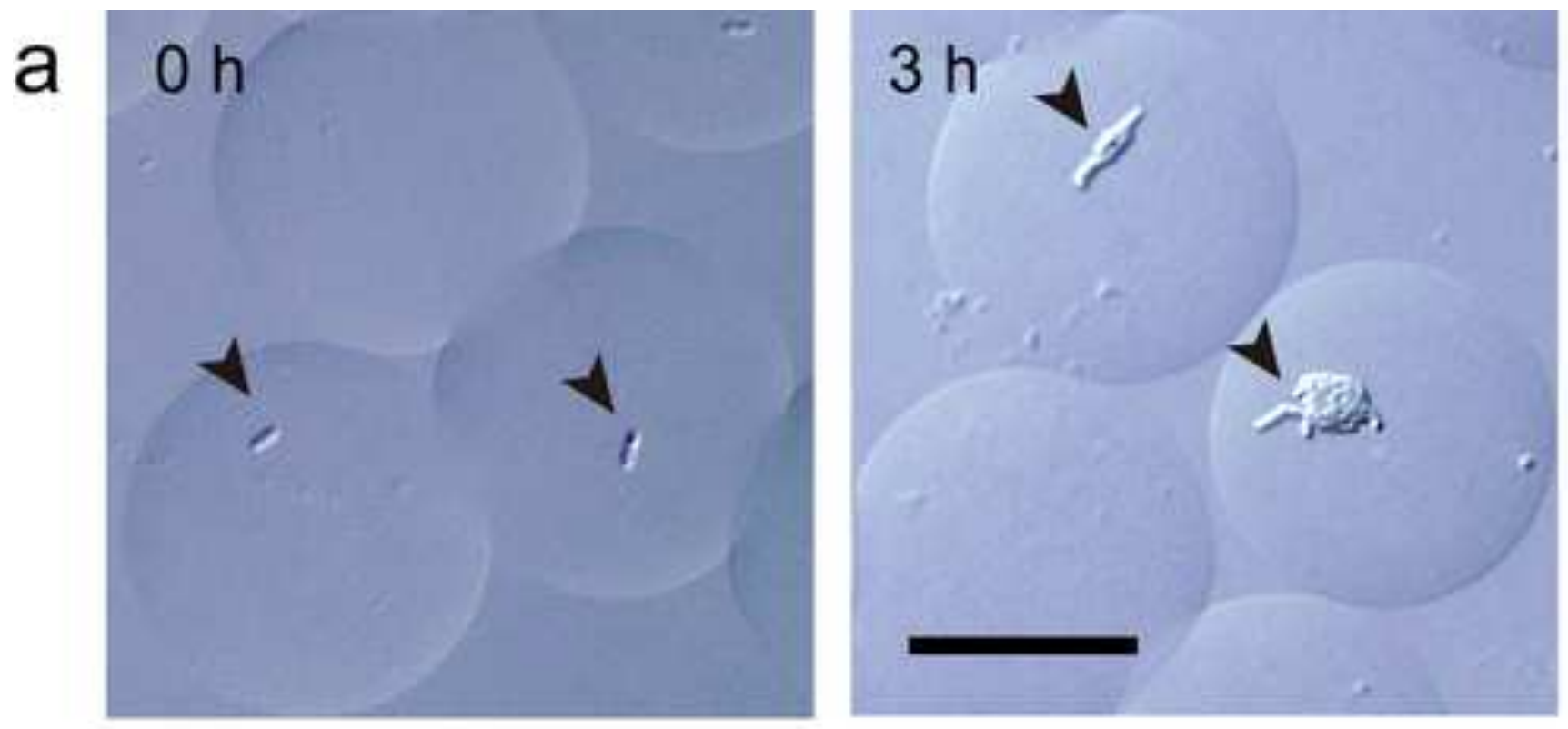

b
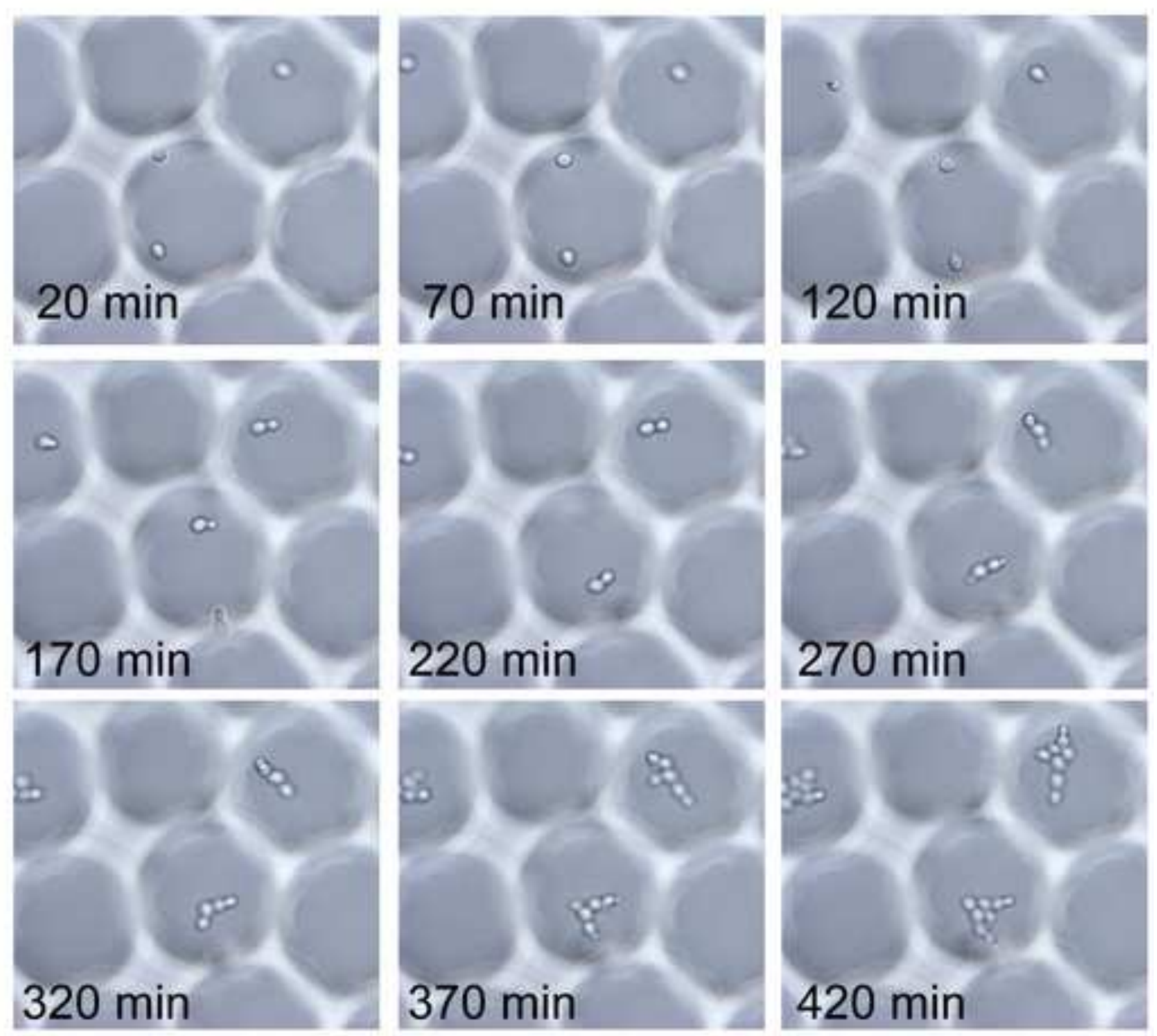
a

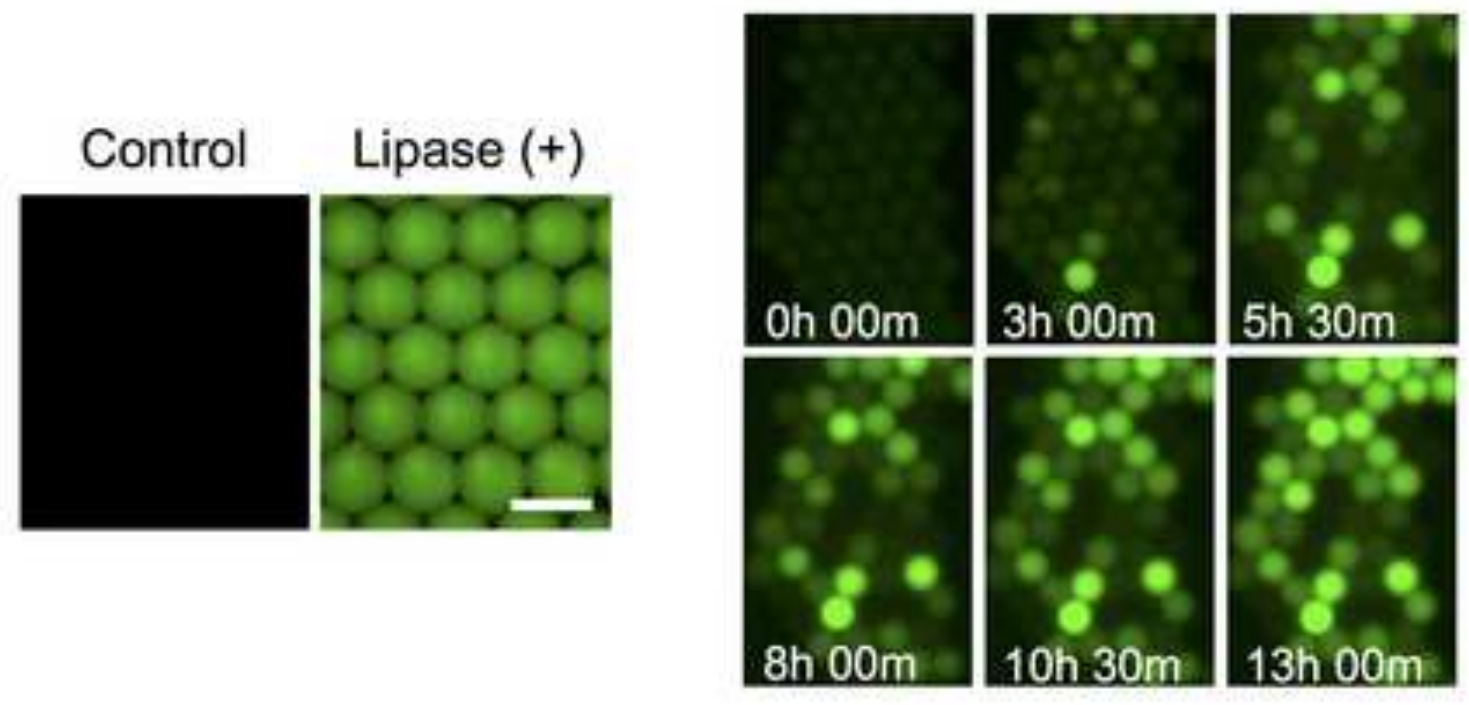

C

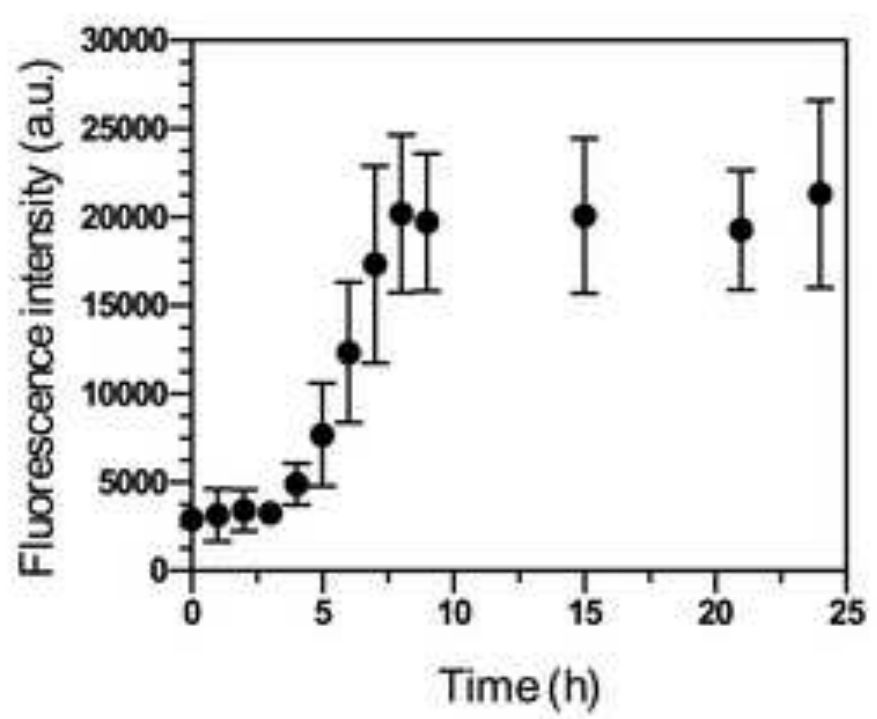

